\title{
Noninvasive Analysis of Synthetic and Decellularized Scaffolds for Heart Valve Tissue Engineering
}

\author{
Nicole Haller, * Trixı Hollweck, Nikolaus Thierfelder, * Julia Schulte, * Jan-Marcel Hausherr, $†$ \\ Martin Dauner, $\neq$ and Bassil Akra*
}

\begin{abstract}
Microcomputed tomography ( $\mu-\mathrm{CT})$ is a nondestructive, highresolution, three-dimensional method of analyzing objects. The aim of this study was to evaluate the feasibility of using $\mu-C T$ as a noninvasive method of evaluation for tissue-engineering applications. The polyurethane aortic heart valve scaffold was produced using a spraying technique. Cryopreserved/thawed homograft and biological heart valve were decellularized using a detergent mixture. Human endothelial cells and fibroblasts were derived from saphenous vein segments and were verified by immunocytochemistry. Heart valves were initially seeded with fibroblasts followed by colonization with endothelial cells. Scaffolds were scanned by a $\mu-C T$ scanner before and after decellularization as well as after cell seeding. Successful colonization was additionally determined by scanning electron microscopy (SEM) and immunohistochemistry (IHC). Microcomputed tomography accurately visualized the complex geometry of heart valves. Moreover, an increase in the total volume and wall thickness as well as a decrease in total surface was demonstrated after seeding. A confluent cell distribution on the heart valves after seeding was confirmed by SEM and IHC. We conclude that $\mu-C T$ is a new promising noninvasive method for qualitative and quantitative analysis of tissueengineering processes. ASAIO Journal 2013;59:169-177.
\end{abstract}

Key Words: tissue engineering, biological heart valve, polyurethane heart valve, homograft, microcomputerized tomography

H eart valve replacement is one of the most common surgical interventions in cardiac health care..$^{1-3}$ The implantation of mechanical or biological prostheses is currently state of the art in this field. ${ }^{4}$ However, both types of implants have several limitations that affect the patient's quality of life. ${ }^{5}$ Biological

From the *Department of Cardiac Surgery, Medical Center Munich University, Munich, Germany; +Chair of Ceramic Materials Engineering, University of Bayreuth, Bayreuth, Germany; and \#Institute of Textile Technology and Process Engineering, Denkendorf, Germany.

Submitted for consideration February, 2012; accepted for publication in revised form November, 2012.

Disclosure: The authors have no conflicts of interest to report.

Reprint Requests: Bassil Akra, University of Munich Hospital, Department of Cardiac Surgery, Laboratory for Tissue Engineering, Marchioninistr. 15, 81377 Munich, Germany. Email: bassil.akra@med. uni-muenchen.de.

Copyright (C) 2013 by the American Society for Artificial Internal Organs

DOI: 10.1097/MAT.0b013e31827db6b6 substitutes tend to degenerate resulting in loss of function, thus indicating reoperation. Mechanical heart valves require a lifelong oral anticoagulation therapy and can be accompanied by severe complications. ${ }^{6-10}$ The field of tissue engineering gives hope to solve the deficiencies of recent heart valve prostheses. The variety of approaches to develop tissue-engineered heart valve prostheses is large. The promising approaches are the seeding of decellularized porcine or human heart valves and the application of synthetic materials such as polyurethane as scaffold materials. ${ }^{11-13}$ Seeding is most frequently achieved using vascular or stem cells ${ }^{14,15}$ Seeding scaffolds with autologous cells has the advantage of thrombogenicity and immunogenicity when implanted. ${ }^{16}$ Decellularization success and seeding efficiency must be visualized in an attempt to develop quality control in tissue engineering. Usual techniques for validating cell adhesion to constructs are scanning electron microscopy (SEM) and immunohistochemistry (IHC). However, these procedures require invasive sampling and are, therefore, useless for later clinical application.

We suggest microcomputed tomography $(\mu-C T)$ as a new high-resolution, noninvasive method for feasibility testing of seeded heart valve. ${ }^{17}$ Computed tomography was introduced into clinical practice in $1972 .{ }^{18}$ This imaging technology enables the generation of three-dimensional images by computer-based stacking of two-dimensional x-ray scans after axial rotation around a sample. ${ }^{19}$ Today CT is used in material testing destruction-free examination of archeological troves, in veterinary medicine, and most of all in human medical diagnostics. ${ }^{17,20-23}$ The spatial resolution of $\mu$-CT is $5-50 \mu \mathrm{m}$; this is up to 66 -fold more precise than the resolution achieved by conventional CT in the clinical setting implementation $(330 \mu \mathrm{m}) .{ }^{24}$ Therefore, the aim of this study was to evaluate the feasibility of using $\mu$-CT scanning as a noninvasive evaluation method for tissue-engineering applications, especially pertaining to heart valve constructs.

\section{Materials and Methods}

\section{Heart Valve Fixation}

The aortic walls of the heart valves were sutured (Supolene suture, Resorba Wundversorgung GmbH \& Co. KG, Nürnberg, Germany) to the base ring of special Teflon fixation units (manufactured in-house). The units consisted of two circular plates (outer diameter $\mathrm{d}_{\mathrm{o}}=4.8 \mathrm{~cm}$, inner diameter $\mathrm{d}_{\mathrm{i}}=2.0 \mathrm{~cm}$ ) connected by four struts (height $\mathrm{h}=4.9 \mathrm{~cm}, \mathrm{~d}=0.6 \mathrm{~cm}$ ) to avoid heart valve deformation during further processing. 


\section{Heart Valve Types and Preparation}

Polyurethane heart valve. Aortic polyurethane heart valves (PUHVs) $(\mathrm{h}=55 \mathrm{~mm}, \mathrm{~d}=18 \mathrm{~mm}$ ) were manufactured by ITV-Denkendorf using a polyurethane spraying technique (patent DE 2806030 C2). Randomly oriented polyurethane (PU) fibers were measured to have diameters between $100 \mathrm{~nm}$ and $550 \mathrm{~nm}$. The fibers formed a sheet with a thickness of $0.3 \mathrm{~mm}$. For seeding purpose, PUHV was $\gamma$-sterilized at $10 \mathrm{kGy}$ according to a certified sterilization procedure.

Homograft. After aortic homograft ( $h=50 \mathrm{~mm}, \mathrm{~d}=19 \mathrm{~mm}$ ) thawing in $56^{\circ} \mathrm{C}$ warm phosphate-buffered saline (PBS) (Biochrom AG, Berlin, Germany), cryoprotectiva were removed by washing once with PBS and once with M199 (Biochrom AG) at $56^{\circ} \mathrm{C}$. The homograft was stored for 24 hours in M199 and was decellularized by treatment of the valve with $0.5 \%$ sodium dodecyl sulfate and $0.5 \%$ sodium deoxycholate (SigmaAldrich Chemie GmbH, Steinheim, Germany) dissolved in PBS for 24 hours at room temperature (RT). This was followed by six 24-hour washing steps in which the valves were washed in PBS at RT to remove cellular remnants and chemicals to which the valves were previously exposed. The homograft was incubated for 24 hours in M199 medium at RT before cell seeding.

Biological heart valve. Glutaraldehyde fixation of porcine heart valve $(\mathrm{h}=45 \mathrm{~mm}, \mathrm{~d}=19 \mathrm{~mm}$, Medtronic $\mathrm{GmbH}$, Meerbusch, Germany) was neutralized by $10 \%$ citric acid (Merck KGaA, Darmstadt, Germany) in distilled water for 5 minutes at RT. For a neutral $\mathrm{pH}$, biological heart valve (BHV) was washed thrice with PBS for 15 minutes. The heart valve was stored in endothelial cell growth medium (Promocell $\mathrm{GmbH}$, Heidelberg, Germany) containing 20\% fetal calf serum (FCS, Lonza AG, Verviers, Belgium) for 1 week at $37^{\circ} \mathrm{C} / 5 \%$ $\mathrm{CO}_{2}$. Decellularization of BHV was analogously performed as described for homograft decellularization.

\section{Cell Isolation and Cell Culture}

Endothelial cells (ECs) and fibroblasts (FBs) were isolated from left-over human saphenous vein segments harvested for coronary artery bypass grafting. Samples were taken from the patients only after their informed consent. Veins were stored in M199 medium at $4{ }^{\circ} \mathrm{C}$ for a maximum of 5 days until further processing. The vein segments were cannulated and washed with M199 medium containing $0.2 \%$ heparin (5000 I.E., Ratiopharm $\mathrm{GmbH}$, Ulm, Germany) and $0.5 \%$ gentamycin $(10 \mathrm{mg} / \mathrm{ml}$, Biochrom AG). For EC isolation, veins were filled with $1 \mathrm{mg} / \mathrm{ml}$ collagenase II (280 $\mathrm{u}$, Worthington Biochemical Corporation, NJ) and incubated for 30 minutes at $37^{\circ} \mathrm{C} / 5 \% \mathrm{CO}_{2}$. After $\mathrm{EC}$ isolation, veins were incubated with $2 \mathrm{mg} / \mathrm{ml}$ collagenase II for 40 minutes at the same conditions for FB isolation. After each incubation step, collagenase cell solutions were rinsed out of the vein, centrifuged for 7 minutes at $500 \mathrm{rpm}$, and cultivated in the respective growth media (endothelial cell growth medium: ECGM; fibroblast growth medium: FGM; PromoCell GmbH, Heidelberg, Germany) in culture flasks $\left(12.5 \mathrm{~cm}^{2}\right.$, Becton Dickinson $\mathrm{GmbH}$, Heidelberg, Germany) at $37^{\circ} \mathrm{C} / 5 \% \mathrm{CO}_{2}$. The medium was partially or completely exchanged every 2-3 days. Cells were passaged at confluency.

\section{Heart Valve Seeding and Cultivation}

Heart valve scaffolds were first seeded with FBs, followed by colonization with ECs. For both cell types, a seeding density of $1.5 \times 10^{6}$ cells $/ \mathrm{cm}^{2}$ was used. To ensure a uniform cell distribution, the seeding procedure was performed in a specially designed rotating seeding device (running phase: 2.5 minutes, holding phase: 30 minutes) for 24 hours at $37^{\circ} \mathrm{C} / 5 \% \mathrm{CO}_{2}$. After FB seeding, the seeded heart valves were cultivated for 7 days at $37^{\circ} \mathrm{C} / 5 \% \mathrm{CO}_{2}$ under static conditions. Medium was exchanged every 2-3 days. After this initial cultivation period, FB-seeded heart valves were analogously seeded with ECs. For further analysis, samples were taken from the supravalvular region of the aortic wall as well as from the valvular leaflets. Samples were taken from native, decellularized, and reseeded valves. The samples from the reseeded valves were taken upon completion of EC seeding.

\section{Phenotypic Cell Characterization}

Phenotypic determination of FBs and ECs was performed by morphologic classification using phase contrast microscopy (Carl Zeiss Mikrolmaging GmbH, Göttingen, Germany). Fibroblasts were recognized by their elongated spindle-like appearance. ${ }^{25}$ Endothelial cells were identified by their typical cobblestone morphology. ${ }^{26}$ Additionally, the cell types were verified by immunocytochemistry. Cells were seeded and cultured on culture slides (eight-chamber; BD Biosciences, Bedford). At confluency cells were fixed and stored in $96 \%$ ethanol (Merck KGaA) at $-80^{\circ} \mathrm{C}$ for a minimum of 48 hours. Slides were washed three times with PBS (Biochrom AG) and endogenous peroxidase activity was subsequently blocked via treatment with hydrogen peroxide (Merck KGaA). Primary antibodies for CD31 (ECs; $0.14 \mu \mathrm{g} / \mathrm{ml}$, Dianova GmbH, Hamburg, Germany) and TE-7 (FBs; $0.67 \mu \mathrm{g} / \mathrm{ml}$, Millipore Corp., BioScience Division, Temecula, CA) were applied, respectively, and the slides were incubated for 30 minutes. After washing, the slides were covered with biotinylated link (HRP Detection System, Biozol GmbH, Eching, Germany) according to manufacturer's protocol and were incubated for 10 minutes. Cells were labeled with HRP streptavidin for 10 minutes and subsequently incubated with AEC-peroxidase substrate (Vector Laboratories, Inc., Burlingame, CA) for another 10 minutes. All steps were performed at RT. Cell nuclei were stained with hemalaun (1:4 in PBS; Merck KGaA). A bright field microscope (Carl Zeiss Mikrolmaging $\mathrm{GmbH}$ ) was used for microscopical evaluation.

\section{Microcomputerized Tomography}

Scanning procedure. For transport and $\mu-\mathrm{CT}$ scanning, heart valve scaffolds were sutured to Teflon units and placed in a custom-fit glass container. Microcomputed tomography scanning of BHV was performed at RT before incubating the biological valve in cell medium by using the original glutaraldehyde-fixation solution, after decellularization and incubation in M199 medium, and finally after seeding and incubation in ECGM. The homograft was scanned at RT after thawing in M199 medium, after decellularization and incubation in M199 medium, and finally after seeding and incubation in ECGM. In the case of PU, scans were performed at RT on a dry scaffold, after seeding and incubating the scaffold for 7 days in FGM, and finally after seeding and incubating the scaffold for 7 days in ECGM. To obtain the necessary high-contrast images, approximately $90 \%$ of the medium was drained from the glass container, and $10 \%$ was left to avoid dehydration of the heart valve scaffolds during scanning. 
Microcomputed tomography analysis allowed a noninvasive analysis of several positions of the scaffold heart valve types. The tomographic investigations were performed using a $\mu-\mathrm{CT}$ machine of the type HR-150-03. The reconstructed complete volume of the heart valve consisted of $2048^{3}$ voxels with an edge length of $35 \mu \mathrm{m}$ and was reconstructed from 1,600 individual $\mathrm{x}$-ray radioscopy images. The detector used was a Perkin-Elmer flat panel detector type XRD 1621-CT3 consisting of $2048^{2}$ pixels. The $x$-ray source used a tungsten target and was powered at $120 \mathrm{kV}$ with a current of $150 \mu \mathrm{A}$, generating a polychromatic $\mathrm{x}$-ray spectrum. The total exposure time was 35 minutes. In addition, a set of region of interest (ROI) measurements were performed to determine the thickness of the cellular coverage. The ROI procedure analyzes a specific small volume of a sample at very high resolution, ignoring the remaining volume. The procedure consists of an off-center measurement, with a focus on one specific region of the volume. A $360^{\circ}$ rotation was performed around the ROI, resulting in high-resolution, high-contrast volumetric images of $9 \mu \mathrm{m}$, allowing the detection of thin cell layers.

For x-ray observation, heart valves do not need to be removed from the sterile container, offering an analysis under sterile conditions.

Image generation. The reconstruction of cross-sections was performed using a reconstruction algorithm developed by the Fraunhofer Institute EZRT, Fürth, Germany. Structural analysis and three-dimensional visualization were carried out using the visualization software VG-Studio Max, version 2.1, with an adapted wall-thickness algorithm developed by the University of Bayreuth. The algorithms used to determine the wall thickness are based on topographic algorithms. The algorithm first determines the exact surfaces of the valve (based on gray-scale gradients of voxels), resulting in two topographic data sets (e.g., inside and outside). For determining the actual thickness at a certain position, both topographic data sets are compared and used to calculate a local minimal distance vector at each position. The vector provides information on the direction as well as the local wall thickness. This approach is applied for each individual CT measurement. Geometric changes in the object are automatically correlated by applying the minimum distance method-any changes in the overall geometry result in a new set of vectors that include the geometric information and the new wall thickness, allowing for a direct comparison of different CT measurements.

The used $\mathrm{x}$-ray tension was adjusted to $120 \mathrm{kV}$ at $150 \mu \mathrm{A}$. The high resolution achieved $(35 \mu \mathrm{m})$ resulted in commendable accuracies of $0.02 \mathrm{~mm}^{2}$ for the determination of total surface area and $0.1 \mathrm{~mm}^{3}$ for the determination of total volume. For our application, total volume was defined as the volume of the complete tissue-engineered structure itself, the lumen of the valve was excluded from this calculation. To determine the thickness of the cellular coverage, regions of the heart valve scaffolds were additionally subjected to an ROI analysis with a resolution of $9 \mu \mathrm{m}$.

\section{Scanning Electron Microscopy}

To detect surface topography of synthetic, biological, and human heart valve scaffolds in native, decellularized, and seeded status, the samples were analyzed by SEM. Specimens were fixed in a solution of $456 \mathrm{ml}$ aqua bidistilled (Ampuwa, Fresenius Kabi Deutschland $\mathrm{GmbH}$, Bad Homburg v.d. H.,
Germany), $0.75 \mathrm{ml} 1 \mathrm{~N}$ hydrochloric acid (Titrisol, Merck $\mathrm{KGaA}), 43.5 \mathrm{ml}$ glutaraldehyde, and $5.65 \mathrm{~g}$ sodium cocodylate trihydrate (Sigma-Aldrich Chemie $\mathrm{GmbH}$ ) at $4{ }^{\circ} \mathrm{C}$ for a minimum of 48 hours. The samples were dehydrated in an ascending ethanol series $(30 \%, 50 \%, 70 \%$, and $95 \%$ ) and subsequently in $100 \%$ acetone. The specimens were dried by critical point drying, sputtered with gold for 180 seconds at $10^{-5}$ mbar, and analyzed using SEM (EVO LS 10; Carl Zeiss Microlmaging GmbH).

\section{Immunohistochemistry}

Immunohistochemical analysis was performed to detect ECs and FBs after seeding as well as to evaluate the native and decellularized biological valves. Samples from native, decellularized, and seeded heart valves were fixed in $4 \%$ formaldehyde (Microcos $\mathrm{GmbH}$, Garching, Germany) for 10 days at $4{ }^{\circ} \mathrm{C}$. Fixed samples were paraffin-embedded and sectioned at $10 \mu \mathrm{m}$. Slices were stained for CD31 (ECs; $0.09 \mu \mathrm{g} / \mathrm{ml}$; Dianova $\mathrm{GmbH}$ ) and TE-7 (FBs; $2 \mu \mathrm{g} / \mathrm{ml}$, Millipore Corp., BioScience Division). Endogenous peroxidase activity was blocked by treatment with hydrogen peroxide and then the samples were incubated with primary antibody overnight at $4^{\circ} \mathrm{C}$. All the following steps were performed at RT. After washing with PBS, the samples were covered with biotinylated link for 10 minutes, and HRP Streptavidin label for 10 minutes according to the manufacturer's protocol (HRP Detection kit, Biozol $\mathrm{GmbH}$ ), followed by chromogen labeling for 10 minutes using AEC peroxidase substrate kit (Vector Laboratories, Inc.). Cell nuclei were stained with hemalaun (1:4 in PBS; Merck KGaA). Controls for nonspecific binding of the chromogen label were performed by excluding the primary antibody. Samples were observed using bright field microscopy (Carl Zeiss Mikrolmaging $\mathrm{GmbH}$ ).

\section{Results}

The evaluation of aortic heart valve in different states (native, decellularized, and seeded) was successfully achieved via $\mu$ CT scanning. To this end, BHV, homograft, and PUHV were tested by $\mu$-CT, with special focus on the effect of seeding on values calculated from $\mu-C T$ data. Microcomputed tomography analysis of homograft aortic heart valves was performed analogously and showed similar results (data not shown).

\section{Topography and Seeding Analysis of BHV and Homograft}

Scanning electron microscopy analysis (Figure 1A-C) of native (A) and in particular of the decellularized (Figure 1B) BHV samples showed randomly orientated fibers resulting in a rough surface. Decellularized BHV demonstrated a confluent cell layer after seeding procedure (Figure 1C). Moreover, typical cobblestone morphology indicated an EC layer. Immunohistochemistry analysis (Figure 1D-F) of decellularized homograft (Figure 1D) did not show the presence of vascular cells. In contrast, reseeded homograft revealed a positive staining for CD31 and TE-7, indicating a confluent coverage with FBs (Figure 1E, brown) and ECs (Figure 1F, brown). Cell nuclei were stained with hemalaun (Figure 1D-F, purple).

\section{Total Volume and Surface Area of BHV}

Microcomputed tomography scanning of heart valve scaffolds was performed as described in the Materials and Methods 


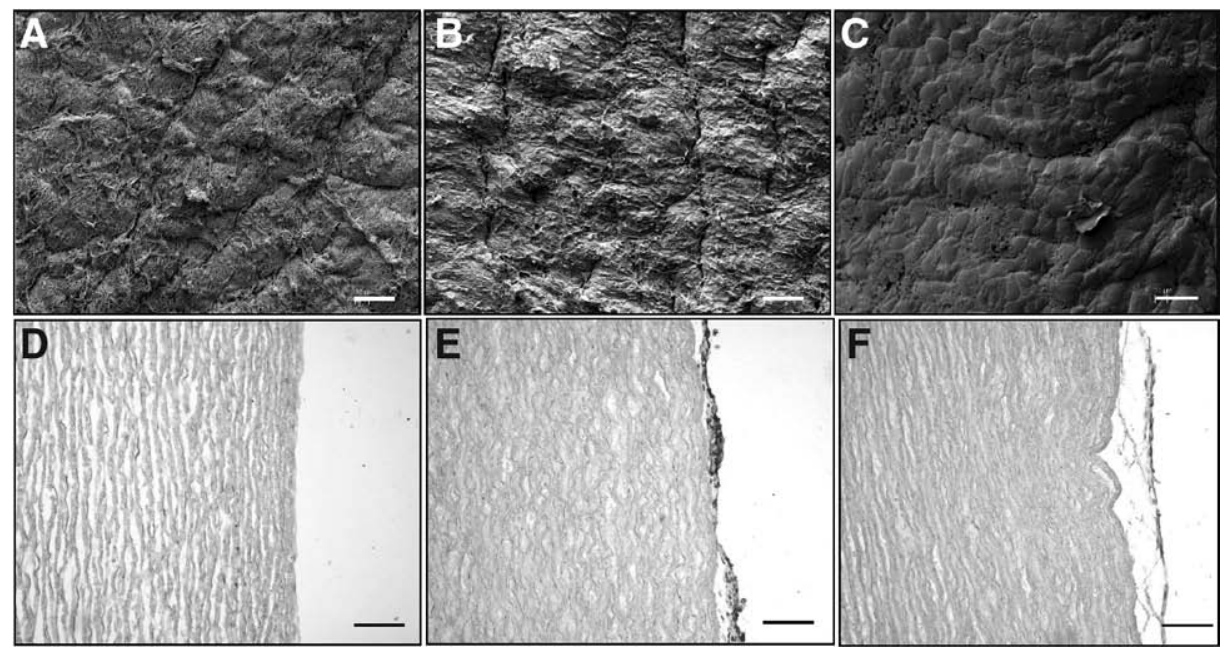

Figure 1. Scanning electron microscopy (SEM) and immunohistochemistry (IHC) analysis of biological heart valve (BHV) and homograft in different states of processing. SEM analysis of native $(\mathbf{A})$ and decellularized $(\mathbf{B})$ samples of BHV showed disordered fibers resulting in a rough surface. In contrast, reseeded samples (C) revealed a confluent endothelial layer. The acellularity of decellularized homograft samples was confirmed by IHC (D). In contrast, stainings reveal various layers of fibroblasts (FBs) (E; TE-7, brown) and endothelial cells (ECs) (F; CD31, brown) after seeding of the homograft. Cell nuclei were stained with hemalaun (D-F, purple). These are representative images of three independent experiments. Scale bars: A, C $=30 \mu \mathrm{m} ; \mathbf{B}=40 \mu \mathrm{m} ; \mathbf{D}-\mathbf{F}=100 \mu \mathrm{m}$.

section. Microcomputed tomography analysis allowed a numerical determination as well as a graphical representation of total volume and surface area. Microcomputed tomography images in Figure 2 show cross- (A-C) and longitudinal sections (D-F) of native, decellularized, and reseeded BHV. A closer look revealed an intense thickening of the valvular leaflets (Figure 2C, arrows) and the aortic wall (Figure 2F, arrows) in the reseeded state.

Quantitative analysis revealed a $0.5 \%$ decrease in total volume and $0.2 \%$ increase in surface area after decellularization of BHV. In contrast, the analysis of seeded BHV after successful decellularization revealed an $8.6 \%$ increase in volume and a $0.3 \%$ decrease in surface area. Single values are summarized in Table 1.

\section{Thickness Alteration of BHV}

Changes in thickness during BHV processing were determined by the comparison of reconstructed cross-sections of the same valve and the same section plane in different states. Figure 3 demonstrates the native (A), decellularized (B), and reseeded $(\mathrm{C})$ states of $\mathrm{BHV}$. The four circular areas represent the Teflon struts of the heart valve fixation unit. At four positions, thicknesses are exemplary determined. The native state of BHV (Figure 3A) revealed a decrease in thickness compared with the decellularized state (Figure 3B). The analysis of the reseeded state (Figure $\mathbf{3 C}$ ) compared with the decellularized and native state shows an increase in thickness at corresponding positions.

\section{Wall Thicknesses of BHV}

Wall thicknesses were volumetrically determined by using tangential analysis of the raw data. The thicknesses of the leaflets (Figure 4A-C) and of the aortic regions (Figure 4D-F) in native, decellularized, and reseeded status are color coded. Different
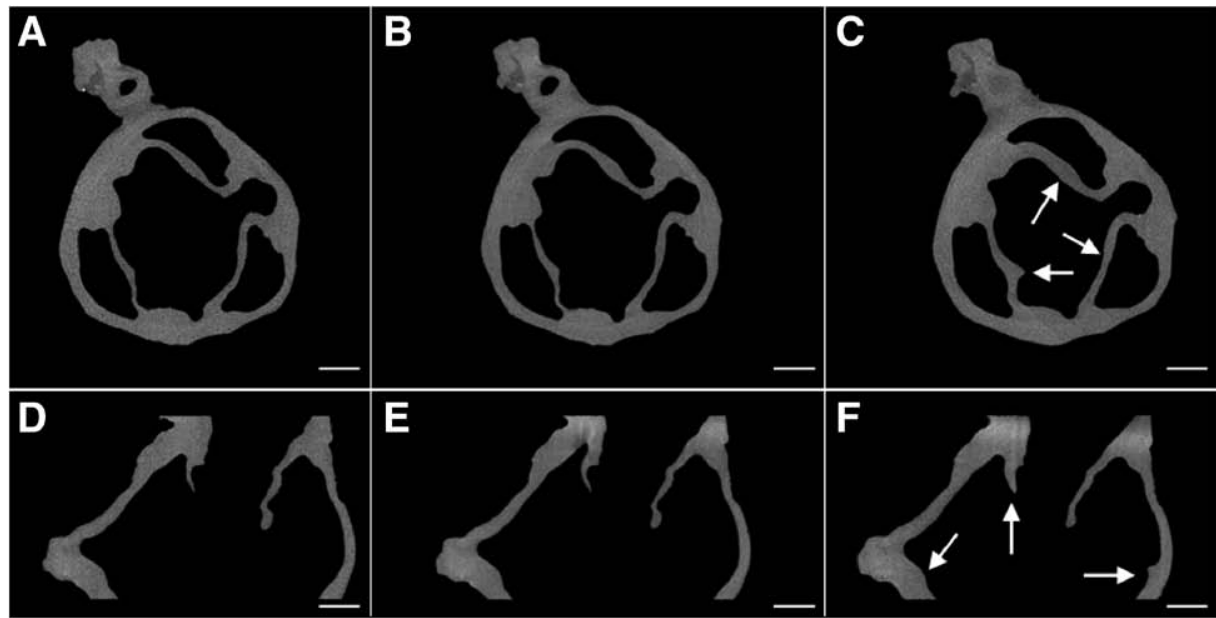

Figure 2. Cross- (A-C) and longitudinal (D-F) sections of native, decellularized, and reseeded biological heart valve from micro-computed tomography scan. Reseeded samples showed a distinct increase in the thickness of the valvular leaflets (C, arrows) and the aortic wall (F, arrows). These are representative images of three independent experiments. Scale bars: $\mathbf{A}-\mathbf{C}=5 \mathrm{~mm} ; \mathbf{D}-\mathbf{F}=10 \mathrm{~mm}$. 
Table 1. Total Volume and Surface Area of BHV in Different States of Processing*

\begin{tabular}{|c|c|c|c|c|}
\hline \multirow{2}{*}{$\begin{array}{l}\text { BHV State } \\
\text { Native }\end{array}$} & \multicolumn{2}{|c|}{$\begin{array}{l}\text { Total Volume } \\
\qquad\left(\mathrm{mm}^{3}\right)\end{array}$} & \multicolumn{2}{|c|}{$\begin{array}{l}\text { Total Surface } \\
\left(\mathrm{mm}^{3}\right)\end{array}$} \\
\hline & 2197 & & 4813 & \\
\hline Decellularized & 2185 & $-0.5 \%$ & 4904 & $+0.2 \%$ \\
\hline Reseeded & 2360 & $+8.6 \%$ & 4775 & $-0.3 \%$ \\
\hline
\end{tabular}

Quantitative microcomputed tomography analysis demonstrated a decrease in volume and simultaneous increase in surface area after decellularization. Reseeding of decellularized BHV resulted in an expansion of volume accompanied with reduction of surface area. These are representative results of three independent experiments. $\mathrm{BHV}$, biological heart valve.

levels of magnification are used for displaying the leaflets than for displaying the aortic regions to improve the visualization of changes in thickness. Increasing levels of thickness are indicated by red, green then blue coloring. Native (Figure 4A) and decellularized leaflets (Figure 4B) as well as native (Figure 4D) and decellularized aortic regions (Figure $\mathbf{4 E}$ ) showed comparable wall thicknesses between states of processing (region specific). In contrast, after seeding a distinct thickening of the leaflet (Figure $\mathbf{4 C}$ ) and the aortic regions (Figure $\mathbf{4 F}$ ) was observed.

\section{Thickness Variance of PUHV}

As described in the Materials and Methods section, the algorithms used to determine the wall thickness are based on topographic algorithms. Changes in thickness of seeded PUHV compared with native status are demonstrated in three-dimensional graphics (Figure 5). Thickness variances are color coded; increasing levels of thickness are indicated by green, yellow, and then pink coloring. Decreasing levels of thickness are by turquoise and then blue coloring. In a vascular view (Figure
5A), a ventricular view (Figure 5B), and a coronal view (Figure $5 \mathbf{C}$ ), leaflets showed a thickening of up to $0.40 \mathrm{~mm}$ (pink). Analysis of the aortic wall revealed a thickening up to $0.16 \mathrm{~mm}$ after seeding (green), with several regions having thickened by up to $0.32 \mathrm{~mm}$ (red) variance.

In addition, Figure 6 demonstrates the cumulated variance distribution of seeded PUHV compared with native PUHV. The histogram correlates the absolute deviation to the surface area and revealed a thickening of up to $0.210 \mathrm{~mm}$ at $90 \%$ of the surface. Ten percentage of the surface showed a thickening between $0.210 \mathrm{~mm}$ and $0.400 \mathrm{~mm}$, proving colonization at every position of the PUHV.

Furthermore, Figure 7 shows ROI measurements of FBseeded (A) and FB- and EC-seeded (B) PU scaffolds. At eight positions in each case, thicknesses are exemplary determined. The different contrast of the scaffold and the cellular coverage clearly demonstrate that a cell layer is available. Moreover, the FB- and EC-seeded (Figure 7B) scaffold revealed a thicker cellular coverage than the FB-seeded (Figure 7A) scaffold, explainable by the presence of EC. Immunohistochemistry analyses support this declaration as well. Staining for TE-7 of FB-seeded (Figure 7C) and staining for CD31 of FB- and EC-seeded (Figure 7D) scaffolds demonstrated the presence of FB (Figure 7C; brown) and a thin endothelial lining (Figure 7D; brown), respectively. Cell nuclei were stained with hemalaun (Figure 7C-D; purple).

\section{Discussion}

Conventional heart valve replacement using synthetic materials or unseeded biological valves is limited by viability, durability, and thrombogenicity. Tissue-engineered heart valves seeded with autologous cells constitute a promising alternative because of their potential antithromobogenicity effect and

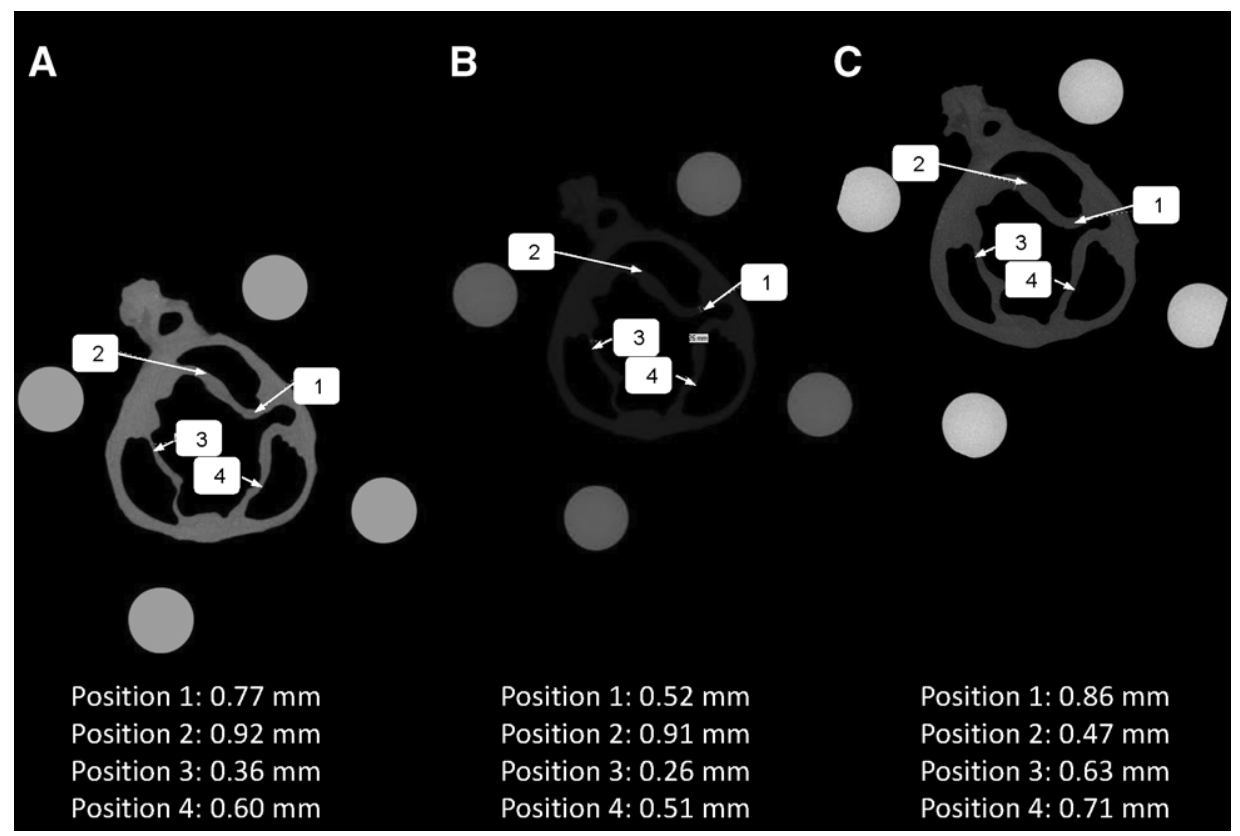

Figure 3. Thickness alteration of biological heart valve (BHV) in different processing states. The comparison of the native (A) and the decellularized (B) state of BHV shows a decrease in thickness at four exemplary positions. In contrast, the analysis in the reseeded state (C) compared with the decellularized and native state revealed an increase in thickness. 

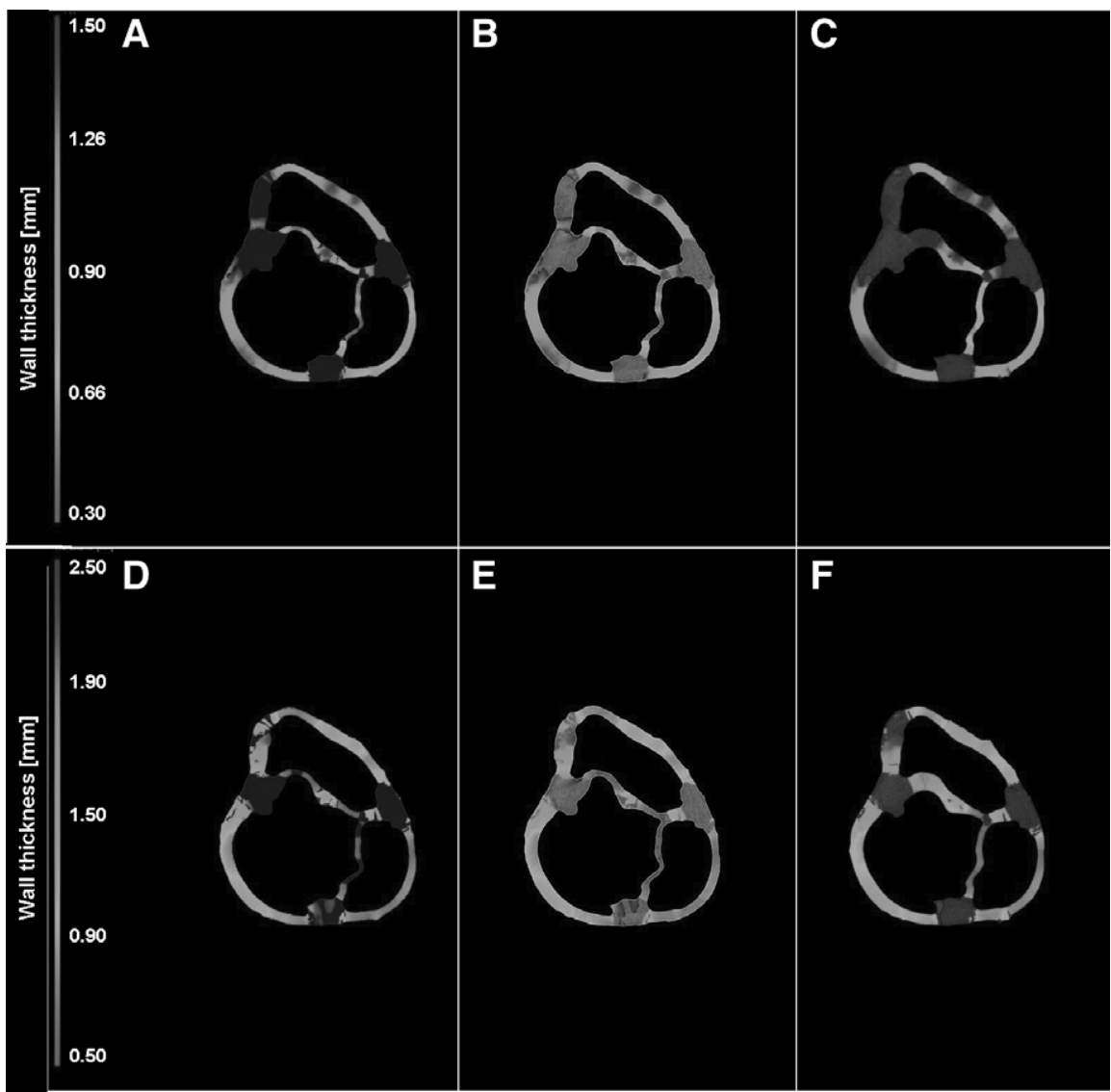

Figure 4. Wall thicknesses of biological heart valve (BHV) in different processing states. Micro-computed tomography analysis virtually generated cross-sections of BHV, illustrating the wall thickness in a color-coded manner. Different levels of magnification are used for displaying the valvular leaflets than for displaying the aortic wall regions to improve the visualization of changes in thickness in the different regions. Native (A) and decellularized leaflets (B) as well as native (D) and decellularized aortic regions (E) showed comparable wall thicknesses between states of processing (region specific). In contrast, reseeding results in a thickening of leaflets $(\mathbf{C})$ and aortic wall regions (F). These are representative images of three independent experiments.

good immunocompatibility. An effective quality control of heart valve implants is indispensable for the preoperative evaluation of tissue-engineered valves planned for implantation.
Despite the successful application of SEM and IHC for the assessment of seeding efficiency, these procedures are inappropriate for quality control of valves produced for implantation

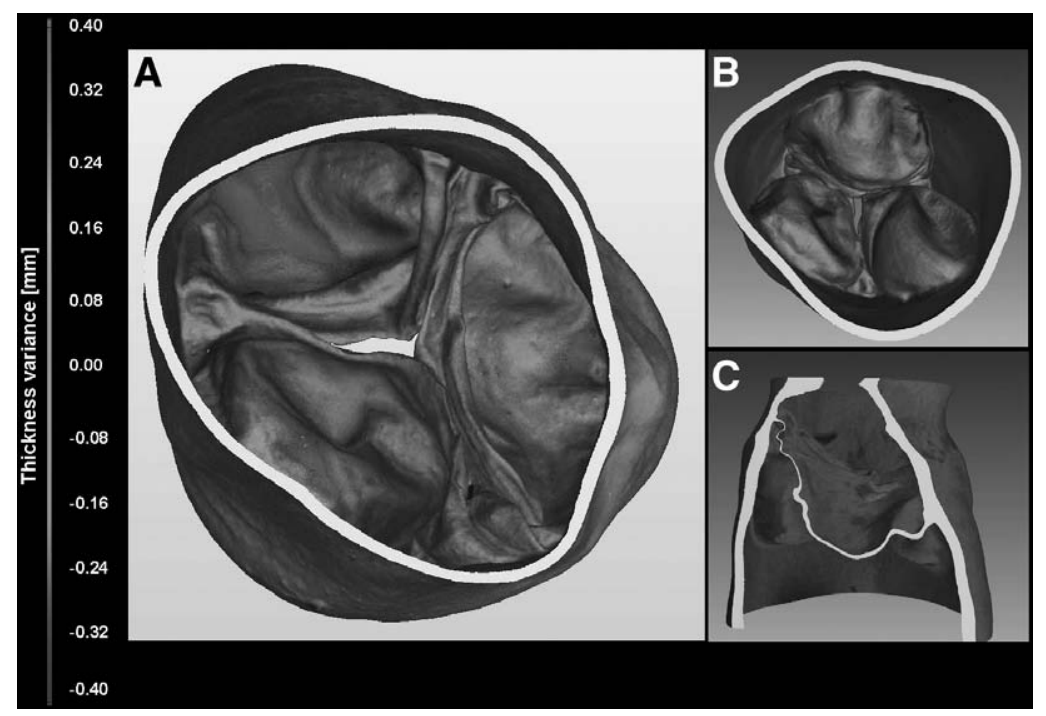

Figure 5. Thickness changes of polyurethane heart valve (PUHV) after seeding. Three-dimensional graphics represent changes in thickness of seeded PUHV compared with native PUHV by color coding. The vascular view (A), the ventricular view (B), and the coronal view (C) reveal a clear thickening of the valvular leaflets and of the aortic wall $(0.40 \mathrm{~mm}$ : pink; $0.32 \mathrm{~mm}$ : red, $\leq 0.16 \mathrm{~mm}$ : green). These are representative images of three independent experiments. 


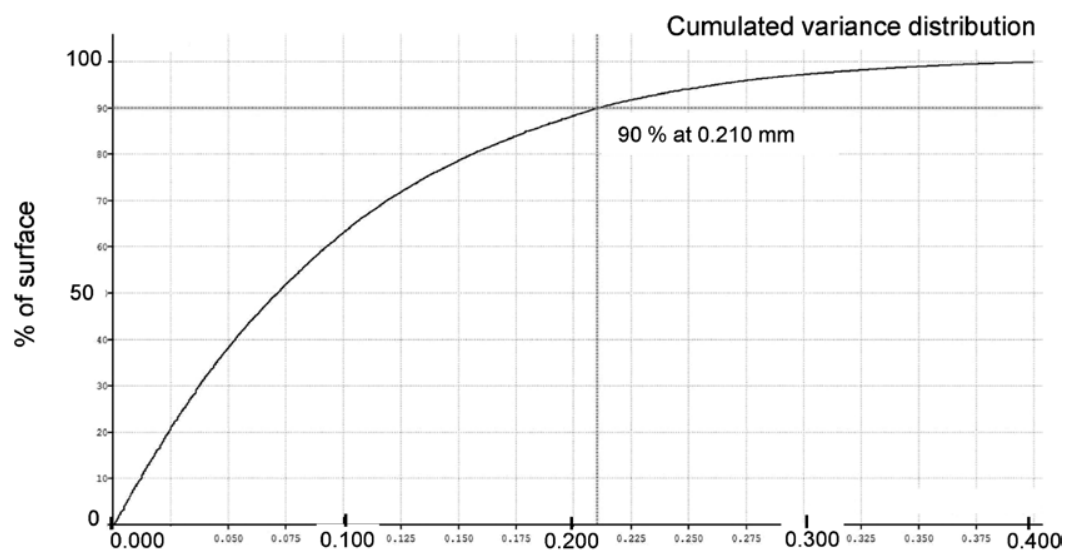

Abs. deviation [mm]

Figure 6. Cumulated variance distribution of seeded polyurethane heart valve (PUHV) compared to native PUHV. Ninety percent of the surface revealed a thickening of up to $0.210 \mathrm{~mm}$. Ten percent of the surface showed a thickening between $0.210 \mathrm{~mm}$ and $0.400 \mathrm{~mm}$, indicating a total colonization of the PUHV. This is a representative histogram of three independent experiments.

because of the invasive nature of this method. Here, we provided evidence that $\mu$-CT scanning is an attractive method for the noninvasive evaluation of tissue-engineered heart valve constructs.

Microcomputed tomography as a modern imaging modality enables the visualization of tissue constructs with highly complex geometries, for example, the meniscus of a knee. ${ }^{27}$
In fact for several years, $\mu$-CT has been a standard tool for biomechanical evaluation and optimization in orthopedic surgery. Jaecques et al. ${ }^{28}$ even examined the postimplantation adaptation of bone tissue by $\mu-C T$. The ability of $\mu$-CT to visualize complex geometries precisely as tested in orthopedics is in line with results generated in this study. We successfully imaged
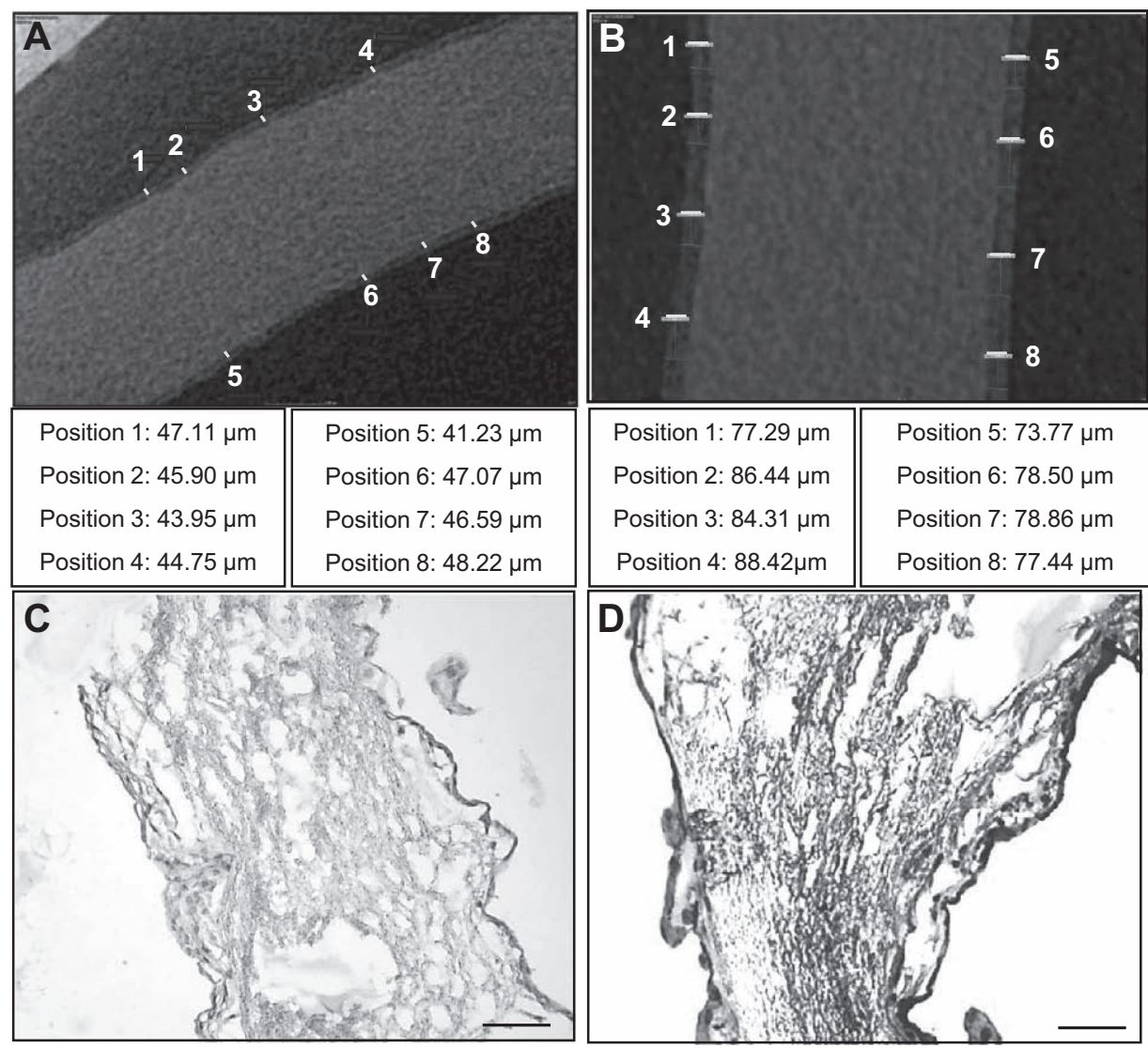

Figure 7. Region of Interest measurements of seeded polyurethane (PU) scaffolds. The thickness of the cellular coverage of fibroblasts (FB)-seeded (A) as well as FB- and endothelial cells (EC)-seeded (B) PU scaffolds were exemplary compared at eight positions. The FB- and EC-seeded (B) scaffold revealed a thicker cell layer than the FB-seeded (A) scaffold, indicating EC presence. Immunohistochemistry analyses also demonstrated a FB layer (C; TE-7, brown) with a thin endothelial lining (D; CD31, brown). Cell nuclei were stained with hemalaun (C-D; purple). Scale bars: $\mathbf{C}=125 \mu \mathrm{m} ; \mathbf{D}=350 \mu \mathrm{m}$. 
the complex structure of our tissue-engineered heart valves. Further studies regarding the effect of $\mu-C T$ on the cell viability should be carried out. Seeded heart valves were not maintained under physiological conditions such as $37^{\circ} \mathrm{C}$ and $5 \%$ $\mathrm{CO}_{2}$ during scanning. These conditions were classified as not required because of the short evaluation time in sterile containers. However, $10 \%$ of media was left in the glass container to avoid dehydration of the heart valve scaffolds during scanning.

We determined that there are many aspects that can be evaluated by $\mu$-CT scanning. Besides the calculation of surface area and total volume, overlaying cross-sections of the valve at different states of processing provides important information about region-specific alterations of the valves' thickness. A deviation of cell layer thickness has been specially detected on the upper side of the cusps of the scaffold. This deviation is probably related to the seeding procedure. Cells are normally added into a seeding cylinder containing the scaffold. This process leads probably to the formation of a cell clump on the upper side of the cusps. It is clearly not a deviation because the three-dimensional images are based on approximately 1,300 images/side and valves were primarily fixed on Teflon support system for stabilization.

The results of REM analysis showed a confluent EC layer on the surface of the BHV. Moreover, the quantitative calculation enabled the definition of the minimum and maximum thickness of the seeded cell layer. This is applicable to decellularized $\mathrm{BHV}$, decellularized homograft, and PUHV, and facilitates the verification of the success of different tissue engineering processes.

Other common methods for precise imaging are highfrequency ultrasound (HFU) and magnetic resonance imaging (MRI) of ferrumoxide-labeled cells. ${ }^{29,30}$ However, HFU and MRI only achieve a maximum resolution of $100 \mu \mathrm{m} .{ }^{30,31}$ In contrast, current $\mu-C T$ scanners provide a resolution of 35 $\mu \mathrm{m}$, thus making $\mu-\mathrm{CT}$ nearly three times more accurate than HFU and MRI. ${ }^{32}$ The algorithm used in this study to determine the surface topography includes a large number of neighboring voxels, resulting in topographic surface information with improved resolution. Typically, the topographic data gained from a 35- $\mu \mathrm{m}$ voxel resolution are accurate to at least a factor of four, providing an accurate topographic resolution of less than $10 \mu \mathrm{m}$. Using ROI technique resulted in very highly detailed images with a $9-\mu \mathrm{m}$ voxel resolution a priori and a posteriori, allowing the detection of thin cell layers. An even higher detailed volume using ROI measurement $(2-\mu \mathrm{m}$ voxel resolution) is possible using a tailored glass container for sterile $\mu$-CT scanning. This would be necessary to reduce the distance between the $\mathrm{x}$-ray tube and the container to the minimum.

Furthermore, HFU is more adequate for use in the imaging of fine tissue structures, ${ }^{29}$ than for creating virtual threedimensional models. Magnetic resonance imaging has several drawbacks. For example, the possibility of the dissolution of ferrum particles after implantation of an imaged construct could put patients at medical risk. ${ }^{33}$ A further disadvantage pertains to the labeling of the cells for imaging. For MRI visualization, cells of tissue constructs must be labeled with magnetic particles such as ferrumoxide. ${ }^{33}$ These foreign molecules may potentially affect cell morphology, viability, and proliferation. If used as a method for preoperative valve evaluation, altering these cellular characteristics could negate the benefit aimed for by implanting a tissue-engineered heart valve. The mentioned MRI linking system furthermore typically makes genetic manipulation of the cells necessary, to provide a counterpart for linking of ferrumoxide particles. Immunoprecipitation labeling systems are alternatives that do not necessitate genetic engineering. ${ }^{34}$ However, such labeling systems result in the distortion of cell morphology and in the alteration of intracellular processes, making this an unattractive approach.

In conclusion, we have demonstrated that $\mu$-CT enables a quantitative calculation and quality control of seeding steps for tissue-engineered heart valve creation. Therefore, we are convinced that $\mu$-CT represents an effective, noninvasive imaging modality for the preoperative evaluation of tissue-engineering constructs.

\section{Author Contributions}

N.H. and T.H. have contributed equally to this study.

\section{Acknowledgments}

The authors thank Medtronic, Inc. for all their support. They also thank Kamil D. Szepanski for the microcomputer tomography measurements and analyses.

\section{References}

1. Goodney PP, O'Connor GT, Wennberg DE, Birkmeyer JD: Do hospitals with low mortality rates in coronary artery bypass also perform well in valve replacement? Ann Thorac Surg 76: 11316; discussion 1136, 2003.

2. Prager RL, Fischer CR, Kong B, et al: The aortic homograft: Evolution of indications, techniques, and results in 107 patients. Ann Thorac Surg 64: 659-63; discussion 663, 1997.

3. Belcher P, Ross D: Aortic root replacement-20 years experience of the use of homografts. Thorac Cardiovasc Surg 39: 117-122, 1991.

4. Filová E, Straka F, Mirejovský T, Masín J, Bacáková L: Tissueengineered heart valves. Physiol Res 58 Suppl 2: S141-S158, 2009.

5. Sacks MS, Schoen FJ, Mayer JE: Bioengineering challenges for heart valve tissue engineering. Annu Rev Biomed Eng 11: 289-313, 2009

6. Chen RDJ, Nihill MD, Denton A, Cooley MD: Early degeneration of porcine xenograft valve in pediatric patients who have undergone apico-aortic bypass. Tex Heart Inst / 9: 41-47, 1982.

7. Hoffmann G, Lutter G, Cremer J: Durability of bioprosthetic cardiac valves. Dtsch Arztebl Int 105: 143-148, 2008.

8. Flanagan TC, Pandit A: Living artificial heart valve alternatives: A review. Eur Cell Mater 6: 28-45; discussion 45, 2003.

9. Rahimtoola $\mathrm{SH}$ : Choice of prosthetic heart valve for adult patients. J Am Coll Cardiol 41: 893-904, 2003.

10. Campos NL, Andrade RR, Silva MA: Oral anticoagulation in carriers of mechanical heart valve prostheses: experience of ten years. Rev Bras Cir Cardiovasc 25: 457-465, 2010.

11. Knight RL, Booth C, Wilcox HE, Fisher J, Ingham E: Tissue engineering of cardiac valves: re-seeding of acellular porcine aortic valve matrices with human mesenchymal progenitor cells. J Heart Valve Dis 14: 806-813, 2005.

12. Elkins RC DP, Goldstein S, Walsh SP, Black KS: Decellularized human valve allografts. Cardiac Bioprostheses 71: 428-432, 2001.

13. Mackay TG, Wheatley DJ, Bernacca GM, Fisher AC, Hindle CS: New polyurethane heart valve prosthesis: Design, manufacture and evaluation. Biomaterials 17: 1857-1863, 1996.

14. Lichtenberg ATI, Cebotari S, Ringes-Lichtenberg S, et al: In vitro re-endothelialization of detergent decellularized heart valves under simulated physiological dynamic conditions. Biomaterials 27: 4221-4229, 2006. 
15. Wu K, Liu YL, Cui B, Han Z: Application of stem cells for cardiovascular grafts tissue engineering. Transpl Immunol 16: 1-7, 2006.

16. Schmidt D, Stock UA, Hoerstrup SP: Tissue engineering of heart valves using decellularized xenogeneic or polymeric starter matrices. Philos Trans R Soc Lond, B, Biol Sci 362: 1505-1512, 2007.

17. Ritman EL: Current status of developments and applications of micro-CT. Annu Rev Biomed Eng 13: 531-552, 2011.

18. Kalender WA: X-ray computed tomography. Phys Med Biol 51: R29-43, 2006

19. Herman GT (ed): Fundamentals of Computerized Tomography: Image Reconstruction from Projection. London, Springer, 2009.

20. Steinhäuser-Andresen S, Detterbeck A, Funk C, et al: Pilot study on accuracy and dimensional stability of impression materials using industrial CT technology. J Orofac Orthop 72: 111-124, 2011.

21. Morigi MP, Casali F, Bettuzzi M, Brancaccio R, D'Errico V: Application of X-ray Computed Tomography to Cultural Heritage diagnostics. Applied Physics A: Materials Science \& Processing 100: 653-661, 2010.

22. Holdsworth DW, Thornton MM: Micro-CT in small animal and specimen imaging. Trends in Biotechnology 20: 34-39, 2002.

23. Kline TL, Zamir M, Ritman EL: Relating function to branching geometry: A micro-CT study of the hepatic artery, portal vein, and biliary tree. Cells Tissues Organs (Print) 194: 431-442, 2011.

24. Mollet NR, Cademartiri F, van Mieghem CA, et al: High-resolution spiral computed tomography coronary angiography in patients referred for diagnostic conventional coronary angiography. Circulation 112: 2318-2323, 2005.

25. Mauch C, Hatamochi A, Scharffetter K, Krieg T: Regulation of collagen synthesis in fibroblasts within a three-dimensional collagen gel. Exp Cell Res 178: 493-503, 1988.
26. Kubota Y, Kleinman HK, Martin GR, Lawley TJ: Role of laminin and basement membrane in the morphological differentiation of human endothelial cells into capillary-like structures. J Cell Biol 107: 1589-1598, 1988.

27. Ballyns JJ, Gleghorn JP, Niebrzydowski V, et al: Image-guided tissue engineering of anatomically shaped implants via MRI and micro-CT using injection molding. Tissue Eng Part A 14: 11951202, 2008.

28. Jaecques SV, Van Oosterwyck H, Muraru L, et al: Individualised, micro CT-based finite element modelling as a tool for biomechanical analysis related to tissue engineering of bone. Biomaterials 25: 1683-1696, 2004.

29. Hanwoo L: High Frequency Ultrasound for Imaging and Characterization of Tissue Engineered Heart Valves. University of Minnesota, Faculty of the graduate school, 2005.

30. Terrovitis JV, Bulte JW, Sarvananthan S, et al: Magnetic resonance imaging of ferumoxide-labeled mesenchymal stem cells seeded on collagen scaffolds-relevance to tissue engineering. Tissue Eng 12: 2765-2775, 2006.

31. Graham KC, Wirtzfeld LA, MacKenzie LT, et al: Three-dimensional high-frequency ultrasound imaging for longitudinal evaluation of liver metastases in preclinical models. Cancer Res 65: 5231 5237, 2005.

32. Engelke K, Karolczak M, Lutz A, Seibert U, Schaller S, Kalender W: [Micro-CT. Technology and application for assessing bone structure]. Radiologe 39: 203-212,1999.

33. Terrovitis J, Stuber M, Youssef A, et al: Magnetic resonance imaging overestimates ferumoxide-labeled stem cell survival after transplantation in the heart. Circulation 117: 1555-1562, 2008.

34. Perea-Saveedra H: Magnetic Vascular Engineering: Development And Validation. Herzogenrath, Germany, Shaker, 2008. 